\title{
L'histoire dans les structures de pointe de la recherche allemande en 2014 : Sonderforschungsbereiche et Exzellenzcluster
}

\section{Pierre Monnet}

\section{OpenEdition}

\section{Journals}

Édition électronique

URL : http://journals.openedition.org/ifha/8017

DOI : 10.4000/ifha.8017

ISSN : 2198-8943

\section{Éditeur}

IFRA - Institut franco-allemand (sciences historiques et sociales)

\section{Édition imprimée}

Date de publication : 31 décembre 2014

ISSN : 2190-0078

Référence électronique

Pierre Monnet, «L'histoire dans les structures de pointe de la recherche allemande en 2014

Sonderforschungsbereiche et Exzellenzcluster », Revue de I'IFHA [En ligne], 6 | 2014, mis en ligne le 31 décembre 2014, consulté le 19 avril 2019. URL : http://journals.openedition.org/ifha/8017 ; DOI : 10.4000/ifha. 8017

Ce document a été généré automatiquement le 19 avril 2019

(CIFHA 


\title{
L'histoire dans les structures de pointe de la recherche allemande en 2014 : Sonderforschungsbereiche et Exzellenzcluster
}

\author{
Pierre Monnet
}

1 Il est dans les fonctions de l'IFHA, dans le droit fil de l'héritage de la MHFA et conformément aux missions de son organe de publication, de fournir aux cercles universitaires, académiques et scientifiques français des clés de compréhension sur la structuration, le financement et les activités de la recherche allemande en Sciences sociales et humaines, et plus singulièrement en histoire. En histoire, cette recherche s'accomplit aussi bien dans les universités, et donc dans les séminaires des facultés correspondantes dont les titulaires et les assistants portent des projets financés sur moyens propres, délégués ou tiers, que par le biais de projets portés par des programmes et des structures transversales et transdisciplinaires, temporaires, communes à plusieurs facultés de la même université, voire à plusieurs universités et pouvant intégrer des instituts de recherche extra-universitaires. Tel est le cas, pour ces programmes transversaux et pluridisciplinaires, des Sonderforschungsbereiche (SFB) d'une part, et des clusters d'excellence de l'autre. Pour les sciences historiques, six de chaque catégorie ont été identifiés et font l'objet de la présente description.

2 Concernant plus particulièrement les Sonderforschungsbereiche (SFB), une précédente équipe du centre en avait fait en 2000 la présentation dans le numéro 36 du Bulletin de la MHFA en décrivant et qualifiant 14 de ces programmes alors actifs dans les sciences et les problématiques historiques (pages 77-101). Depuis, non seulement 14 années ont passé, un délai qui pourrait à lui seul justifier une nouvelle présentation, mais le paysage scientifique et universitaire allemand, comme son homologue français, a subi un remembrement profond de son organisation, de ses financements, de la temporalité et de l'actualité de ses thématiques de recherche. En effet, depuis une quinzaine d'années, le 
processus de Bologne (L/M/D ou 3/5/8), la déclaration de Lisbonne sur la compétitivité du savoir, la crise des finances publiques, la réarticulation des rapports entre recherche publique et recherche privée, les initiatives dites d'excellence, l'importance toujours croissante accordée à l'évaluation, au classement, à la comparaison des universités et des projets de recherche, des chercheurs et de leurs publications, à la capacité des équipes à rassembler des fonds ont profondément modifié la carte des savoirs et des lieux de savoir ${ }^{1}$ . On consultera, pour mieux prendre connaissance de ces réformes et de ces nouvelles structures, le guide de la recherche intitulé « Faire de l'histoire en Allemagne » publié en 2011 sur la plate forme électronique Perspectivia.net ${ }^{2}$. Par ailleurs, les renouvellements inévitables et souhaitables intervenus dans les intérêts, les méthodes, les techniques et les technologies des historiens ont abouti à faire émerger de nouveaux terrains et de nouvelles enquêtes. Il a donc semblé opportun de proposer une présentation concise mais précise des $6 \mathrm{SFB}$ et des 6 clusters d'excellence qui ont paru présenter le plus grand intérêt par leur thématique et leur structuration pour l'avancée de nouvelles problématiques en histoire et pour leur capacité à susciter des coopérations internationales, notamment avec les chercheurs français et francophones.

3 Comme les Graduiertenkollegs (écoles doctorales) ${ }^{3}$, les SFB sont financièrement portés en Allemagne par la DFG ou Deutsche Forschungsgemeinschaft ${ }^{4}$. La DFG est une organisation de droit privé, créée en 1920, financée par le gouvernement fédéral et les régions (Länder), fondée sur le principe de l'autogestion de la recherche et du soutien à des projets limités et évalués dans le temps. Au total, 37 formats de soutien existent (prix, académies, bourses, programmes...) parmi lesquels les SFB, les écoles doctorales et les clusters d'excellence forment les outils les plus lourds et les plus structurants. Les SFB sont conçus comme des programmes de recherche transdisciplinaires à moyen voire long terme (12 ans), réunissant au sein d'une université plusieurs Fachbereiche ou facultés, et mêlant les différents types d'exercice, de rencontres, de publications, de valorisation des résultats dans le souci de nouer recherche et formation à la recherche. Les SFB peuvent également, si besoin est, s'ouvrir à des organismes de recherche extra-universitaires, tels les Instituts Max-Planck, ou bien faire coopérer plusieurs universités d'une même aire régionale (programme Transregio). En 2014, la DFG soutient toutes disciplines confondues 245 SFB pour un budget total de 610 millions d'euros (soit $21 \%$ du budget global de la DFG) 5.13 nouveaux ont vu le jour en 2014. La répartition par grand champ thématique fait état de $25 \mathrm{SFB}$ en sciences sociales et humaines (10\% environ du total), 47 pour les sciences de l'ingénieur (environ 20\%), 70 pour les sciences naturelles (28\%) et 103 (42\%) pour les sciences du vivant.

Les clusters d'excellence sont venus compléter ce dispositif à la faveur de l'initiative d'excellence allemande initiée en 2005 et lancée pour la première phase en 2006-2011 (la seconde est en cours de 2012 à 2017). Les deux phases ont au total représenté un investissement supplémentaire et sélectif de 4,6 milliards d'euros sur la période 2006-2017, répartis en trois lignes de soutien : «concept d'avenir » pour une université dans son ensemble (pourvu qu'elle abrite par ailleurs à la fois un cluster d'excellence et une école doctorale d'excellence, 9 ont été distinguées dans la première phase et 11 dans la seconde), «école doctorale » pour la formation doctorale d'une université (40 pour la première phase et 45 dans la seconde) et «cluster " sur des thématiques transversales faisant collaborer les facultés, les professeurs, les laboratoires et les jeunes chercheurs (doctorants et postdoctorants). Chaque cluster était doté dès 2006 de 6,5 millions d'euros par an. 37 clusters ont été distingués dans la première phase pour une durée allant 
jusqu'en 2010 ou 2011 (5 ans). Lors de la seconde phase, en 2012, 43 clusters ont été installés, soit par renouvellement d'un cluster précédent, soit par création, de nouveau pour une durée de 5 ans $^{6}$. Les universités d'accueil des clusters de la première phase sont: Aix-la-Chapelle, Berlin, Bielefeld, Bonn, Brême, Cologne, Constance, Darmstadt, Dresde, Erlangen, Francfort-sur-le-Main, Fribourg, Giessen, Göttingen, Hambourg, Hanovre, Heidelberg, Karlsruhe, Kiel, Munich, Münster, Sarrebruck, Stuttgart, Tübingen. Hormis la capitale fédérale et l'exception dresdoise, aucune université des nouveaux Länder de l'Est n'en accueille et une forte concentration se signale dans les régions de l'Ouest et du Sud. La seconde phase a sélectionné sans grand bouleversement de la carte des clusters établis dans les universités de Aix-la-Chapelle, Berlin, Bielefeld, Bochum, Bonn, Brême, Chemnitz, Cologne, Constance, Dresde, Düsseldorf, Erlangen, Francfort-surle-Main, Fribourg, Giessen, Göttingen, Hanovre, Heidelberg, Kiel, Mayence, Munich, Münster, Oldenbourg, Sarrebruck, Stuttgart, Tübingen. Parmi eux, 6 relèvent des sciences sociales et humaines (un peu moins de 15\%), dont une seule création quand les 5 autres existaient déjà en 2006/2007; 11 relèvent des sciences naturelles (un peu plus de 25\%) ; 17 des sciences du vivant (un peu plus de 40\%) et 9 des sciences de l'ingénieur (20\%).

\section{NOTES}

1. Voir Béatrice Durand/Stefanie Neubert/Dorothee Röseberg/Virginie Viallon, Étudier en France et en Allemagne. Approche comparée des cultures universitaires, Lille: Presses Universitaires du Septentrion, 2007.

2. Falk Bretschneider/Mareike König (dir.), Faire de l'histoire en Allemagne. Un guide pour les jeunes chercheurs français, 2011, voir notamment Falk Bretschneider/Bernd Klesmann/Rahul Markovits, «Universités, instituts de recherche extra-universitaires et associations », http:// www.perspectivia.net/content/publikationen/scholar-guide/histoire-en-allemagne/ bretschneider-klesmann-markovits_structures (consultation le 16 juillet 2014, comme pour tous les autres sites indiqués dans la présente introduction).

3. Voir la liste: http://www.dfg.de/foerderung/programme/koordinierte_programme/ graduiertenkollegs/index.html.

4. http://www.dfg.de/.

5. Voir la liste : http://www.dfg.de/foerderung/programme/listen/index.jsp?id=SFB.

6. Voir la liste: http://www.dfg.de/foerderung/programme/exzellenzinitiative/ exzellenzcluster/gefoerderte_projekte/index.html. 


\section{AUTEUR}

PIERRE MONNET

(EHESS/IFHA) 